\title{
RADIATION-INDUCED VOLTAGE ON SPACECRAFT INTERNAL SURFACES
}

\author{
A. R. Frederickson \\ Space Physics Division \\ USAF Phillips Laboratory \\ Hanscom AFB, MA 01731-3010
}

\begin{abstract}
The charging of surfaces of insulators beneath the thermal control blankets on spacecraft has been numerically simulated. Such insulators are found on wiring harnesses, bulkhead feed-throughs, component mounting hardware, tiewraps, thermal sensors, etc. These insulators are shielded from the low energy space plasma by the grounded solar blankets. It is determined that the surfaces of these insulators rise to many tens of $\mathrm{kV}$ in the Earth's electron belts unless sufficient conduction to the spacecraft frame is established. Secondary electron emission is not sufficient to hold the potential to low values. The dependence of charging upon insulator thickness, electron energy spectra, and material properties is investigated. This phenomena is posited as an explanation for the Spacecraft Anomalies seen on many spacecraft, and on the CRRES satellite in particular.
\end{abstract}

\section{INTRODUCTION}

Limited space testing results [1] and ground testing results [2] indicate that thick insulators internal to a grounded conducting cavity and exposed to penetrating electrons may not charge to more than a few hundred volts. This fact is inferred [1], not confirmed, from the magnitude of the discharge pulses which insulators have created during both space and ground testing. Both in space [1] and in ground tests [2] where the radiation partially simulated the space conditions, the discharge pulses were so small that the surface voltage dropped less than 100 volts during the pulse. Yet ground testing with tens of $\mathrm{keV}$ electron beams [3] which charge the surfaces to multi-kV potentials provides discharge pulses that cause the surface potential to drop by nearly the full surface potential, $>10 \mathrm{kV}$. Space radiation is capable of charging the surfaces to multi-10keV potentials so one wonders if large discharge pulses are occurring in space similar to those in, say, $25 \mathrm{keV}$ electron beam tests. This paper investigates the voltages developed on insulated surfaces inside spacecraft.

The relationship between surface voltage and pulse magnitude is as follows. Based on the scaling laws of Balmain et al [3], and on laboratory measurements with controlled surface voltages [4], one presumes that many of the discharges nearly fully deplete the surface voltage on the insulators. Since we measure the total charge which flowed in the pulse discharges, and we know the capacitance of the insulated surfaces to ground, then we know the change in surface voltage resulting from the discharge pulses. If one begins with surfaces well above 100 volts, then the discharge process generates a plasma which nearly fully depletes the surface voltage [4]. Of course it is likely that some pulses discharge only a small fraction of the surface voltage, but lab tests find that a large fraction of pulses discharge nearly the entire surface voltage. Why have we seen only small pulses in the limited space-like tests to date? Could we see much bigger pulses in future space tests? This paper investigates the state of our knowledge to find that there is a serious gap between our basic concepts and the limited space experience. Our basic concepts hint that much larger pulses are possible, well beyond those seen to date.

We would like to know if the experience to date is indicative of most cases for spacecraft in space. Will surfaces inside the spacecraft never charge to more than a few hundred volts relative to the spacecraft frame? If so, then we need only engineer the components of the spacecraft for pulses which do not exceed a few hundred volts. In this paper we use existing tested software to estimate the surface voltages on internal insulators. This software and its generalized calculations are intended to scope our theoretical knowledge of the phenomena. Hopefully the information gained from this study will help us to design better spacecraft.

Reasonably complete 3-D computer codes [5] are available to determine the surface voltages on the outside of a spacecraft. Such codes are still not able to make highly accurate prediction because of two effects, the effects of the Earth's magnetic field on electron trajectories and the effects of the space environment on the secondary electron yield from spacecraft surfaces. The various surfaces of the satellite mutually interact via both the space plasma and the emitted low energy electron intermediaries. The 3-D computer codes are an impressive computing capability which tracks the motions of the incident as well as the intermediate particles.

Similar phenomena will go on inside a satellite where the dominant intermediaries are the secondary electrons and the conduction currents. Since the dominant charging radiation is the penetrating high energy (HE) electrons [1], the problem can be simplified by developing worst case estimates. Such a point of view is taken in this paper; we need not consider the full 3-D complexity when scoping this new problem area. The low energy space plasma (electrons 
and ions) is assumed to not penetrate into the spacecraft.

\section{THE SIMPLIFIED 1-D GEOMETRY}

Fig, 1 describes the simplest geometry. It is a geometry that has been tested in space and on the ground so that we may compare it to real data $[1,2]$. The computer code, NUMIT, solves for the full time dependent electric fields, voltages, and currents in this 1-D geometry. NUMIT has been compared to laboratory tests of insulator charging under high energy (HE) irradiations with good success $[6,7]$. A subroutine within the code is based on monte carlo and experimental electron transport data, and predicts the dose, penetration and stopping of the incident space electrons from $100 \mathrm{keV}$ to $10 \mathrm{MeV}$. However it is limited to electrons normally incident on the outside of the spacecraft, the condition in the laboratory tests [2].

SPACECRAFT FRAME

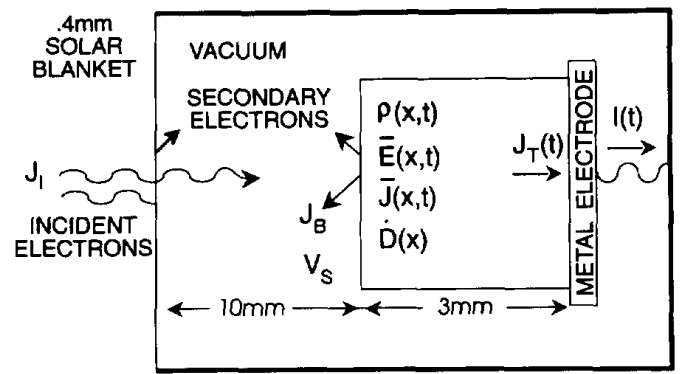

Fig. 1. The Geometry Which Simulates Insulators Beneath Spacecraft Blankets.

\section{THEORY}

\section{A. General Theory}

NUMIT performs the following modelling in 1-D. Refer to Fig. 1. Electrons penetrate the shield and form the incident high energy electron current, $\mathrm{J}_{\mathrm{I}}$. These electrons penetrate into the insulator, in the $x$ direction, forming a portion of the current $J(x, t)$ inside the insulator. For thick insulators most of the incident electrons are stopped inside the insulator. Most electrons which penetrate the insulator, $\mathrm{J}_{\mathrm{T}}$, are stopped in the conductive material upon which the insulator is mounted. Thin insulators, less than $0.1 \mathrm{~mm}$ will experience nearly full penetration by the high energy space flux. The accumulation of charge in the insulator, $\rho(x, t)$, produces electric fields, $E(x, t)$. The electric fields combined with the electron hole pairs generated by both radiation and thermal processes produce conduction currents in the insulator that also contribute to the total current $J(x, t)$. The conduction currents are strongly controlled by the dose, $D(x)$, created in the insulation by the high energy electrons. The divergence of the conduction currents also contributes to $\rho(\mathrm{x}, \mathrm{t})$. Some of the high energy electrons are scattered back from the insulator forming $J_{B}(t)$. Low energy secondary electrons are emitted from both the insulator surface and the satellite skin and contribute to $J_{B}$ and $J_{1}$ respectively. The motion of all of the electrons along with the resulting displacement current, $\mathrm{dE} / \mathrm{dt}$, produce currents, $I$, in the conducting materials behind and near the charging insulator. It is assumed that the spacecraft frame and outer surface are held near space plasma "ground" potential by photoelectron emission and by collection of currents from the low energy plasma. All of these terms are self consistently calculated by the computer code NUMIT for 1$\mathrm{D}$ insulators. For this problem we limit consideration to cases where the electric fields are small enough that their effect on the motion of the HE electrons is negligible.

The surface voltage is determined as the spatial integral of the electric field from ground to the insulator surface. If the total incident electron current, $J_{I}$, is larger than the total backdirected electron current, $\mathrm{J}_{\mathrm{B}}$, plus transmitted current, $\mathrm{J}_{\mathrm{T}}$, then the insulator surface will charge negatively. It will continue to charge negatively to high voltage as long as the net current to the insulator remains negative.

In this paper we address the following question. As the electric fields build up, does the surface voltage continue to increase with time, or are there some limiting phenomena which allow for leakage of the current before the surface voltage rises too high. For typical insulators, voltages of 1 $\mathrm{kV}$ to $10 \mathrm{kV}$ would be beyond design margins and are to be avoided. The computer runs were not continued beyond the time when tens of $\mathrm{kV}$ are achieved. For the space electron spectra, tens of $\mathrm{kV}$ will not substantially alter either the incident or the penetrating high energy electron current.

\section{B.Important Parameters}

A number of parameters will be critical to this modelling. They include dark conductivity, radiationenhanced conductivity, delayed radiation-enhanced conductivity, high field enhanced conductivity, electron backscatter, secondary electron emission and field enhanced secondary electron emission. Only backscatter and secondary emission are parameters which we can know with reasonable assurance at the beginning of the study. The other parameters will be handled in a worst case way.

For most of these studies the coefficient of radiationinduced conductivity will be assumed to be $1 \times 10^{-17} \mathrm{sec} / \mathrm{ohm}$ $\mathrm{cm}$-rad. The dark conductivity will be assumed at an extremely low value of $1 \times 10^{-19}$ per ohm-cm. Hopefully this value corresponds to the lowest value possible for insulators exposed for years to high vacuum in space. These are felt to be worst cases. Handbook values of insulator resistivity are misleading. They are for atmospheric environments with electrodes on the material. Even brief exposure to vacuum will raise the dark resistivity of many insulators by 
orders of magnitude. The best way to check this is to test in the space environment. Testing in vacuum chambers with electron beams has almost always shown me that the dark resistivity is often at $10^{18} \mathrm{ohm}-\mathrm{cm}$ even when handbook values indicate two orders of magnitude less. The electron beam tests measure the resistivity by monitoring the time rate of decay of a specimen previously charged by brief exposure to the electron beam. This is the best test for our space materials. Normal atmospheric insulator testing uses high electric fields with electrodes on the sample thereby producing enhanced conductivity by the method of measurement itself.

The nominal secondary electron yield is determined as follows. The yield $(.25)$ at $10 \mathrm{keV}$ is obtained from figure 2 (which is accidently labeled fig. 4) in Burke [8]. Since the universal yield curve is actually based on the fact that yield is proportional to dose near the surface, we determine the surface dose due to $10 \mathrm{keV}$ electrons. This results in a secondary electron yield ( $\mathrm{SeY})$ current density of $\mathrm{SeY}=$ $1 \times 10^{-13} \times$ dose rate, in amperes $/ \mathrm{cm}^{2}$. Dose rate is in units of rads per second. This relation between secondary electron emission current density and surface dose rate is assumed to hold approximately for all the materials. An alternative derivation is to assume eq. 3 in Ref. 9. One obtains exactly the same emission current density if the sample-specific parameter $\Delta x / \epsilon=0.01$, a most reasonable value $[8,9]$.

Since the computer program, NUMIT, calculates dose directly one is able to determine the secondary electron current density internal to the program which keeps track of the current flows. Thus the electric fields and spacecharge densities reported here explicitly contain the effects of secondary electron emission.

\section{Typical Space Conditions}

For all of these calculations the high energy (HE) electron, $>100 \mathrm{keV}$, flux intensity is assumed to be $10^{-12}$ amperes per square $\mathrm{cm}$. This corresponds roughly to high levels in the electron belts of the Earth. The calculations are performed primarily for the insulator geometry of Fig. 1. The thermal control blanket is simulated by a $0.4 \mathrm{~mm}$ thick layer which is held at or near frame potential. The results do not appear to depend strongly on the thickness of the blanket as long as it is thin enough to allow sufficient electrons to bombard the internal insulators.

Radiation-induced gas ionization is negligible. The ionized ambient gas current density is roughly 8 orders of magnitude smaller than the incident HE electron current for a $1 \mathrm{~cm}$ wide gap in the vacuum space where the density is assumed to be $1 \times 10^{-11} \mathrm{~g} / \mathrm{cm}^{3}$. However, if we hold the size of our sample constant but increase the enclosure volume by 8 orders of magnitude, then the gas ionization could hold the surfaces near the frame potential. This is analogous to developing a plasma sheath inside the satellite cavities.
Thus small samples inside a large satellite cavity might have less problem with surface charging due to conduction in the ionized ambient. It is important to note that the ambient is partially ionized by the penetrating radiations.

The electron current density which is sufficient to cause charging usually depends on the dark conductivity of the thickest insulator, but dark conductivity is a parameter which is unknown in space service after years of exposure to high vacuum and radiation. Our simulations assume an estimated worst case value for dark resistivity; one can not depend on this value but must determine the proper value for the material at hand. To my knowledge, this has not been done for real satellite materials in situ.

To prevent dangerous charging, the product of $\mathrm{HE}$ electron current density $\left(\mathrm{A} / \mathrm{cm}^{2}\right)$ times dark volume resistivity (ohm-cm) times insulator thickness $(\mathrm{cm})$ should be held to less than 50 volts by judicious choice of dark conductivity. This fact is elementary and need not be simulated in the study. Further safety is gained by the use of thin insulators wherein the radiation-induced conductivity (RIC) enhances the leakage all the way through the insulator. The RIC can be depended upon to prevent dangerous charging only when it increases the conductivity sufficiently to prevent surface charging above 50 volts. The value of RIC included in the simulations is $1 \times 10^{-17} \mathrm{sec} / \mathrm{ohm}-$ $\mathrm{cm}$-rad and is based on short term ground measurements. RIC during long term space service is an unknown parameter and it is unwise to assume a value to be correct a-priori.

\section{RESULTS}

Both space spectra and ground based testing spectra are considered below. Thus one may compare ground tests to this theory, and also predict space results. Ground testing will be necessary in order to validate our ideas.

\section{A. $1 \mathrm{MeV}$ Normal Incidence.}

Figure 2 summarizes a study for ground tests using monoenergetic electrons normally incident at $1 \mathrm{MeV}$ upon the cover. All curves include correct high energy backscatter data which is explicit in NUMIT and which is not significantly affected by the electric fields. The highest curve assumes, for comparison purposes, that there is no secondary electron emission (NO SeY) from the material. The other curves are for a nominal level (SeY) of secondary electron emission [8] and for secondary electron emission enhanced by factors of 10,15 and 20 over the most probable value for the sample. For this case one requires a factor of 20 enhancement in secondary yield in order to prevent unacceptable surface voltage. Note that the first four curves continue to increase beyond the time plotted on Fig. 2, but that the curve with the highest secondary electron yield (20X SeY) remains near zero volts forever. 


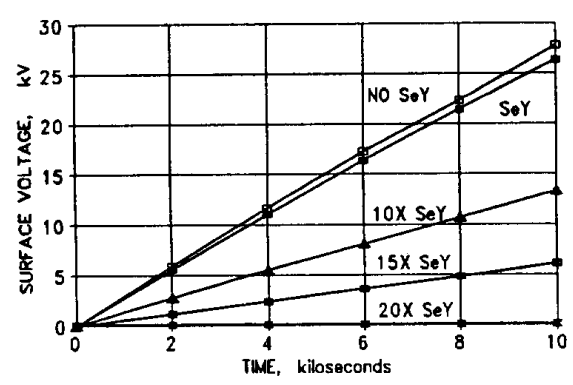

Fig. 2. Surface Voltage by Normally Incident 1 $\mathrm{MeV}$ Electrons.

\section{B. Normal Incidence Space Spectra.}

Actual space spectra have the approximate form $J(E)=E^{-N}$ where $E$ is the incident electron energy. Increasing $N$ has the effect of increasing the surface dose, and therefore the secondary electron emission current if the $\mathrm{HE}$ electron current density is held constant. Figs. 3,4 show the results for $\mathrm{E}^{-4}$ and $\mathrm{E}^{-6}$ spectra. Both spectra are commonly seen in space. With the $\mathrm{E}^{-4}$ spectra the secondary electron yield must be ten times nominal in order to hold the surface at ground potential. The $\mathrm{E}^{-6}$ spectra requires a secondary electron yield to be roughly seven times nominal in order to hold the surface at zero volts.

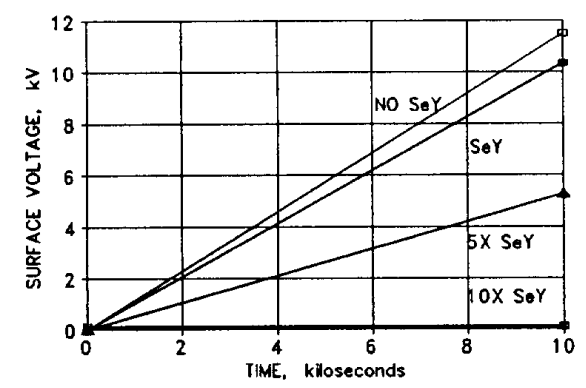

Fig. 3. Surface Voltage by Normally Incident $E^{-4}$ Spectra.

\section{Semi-Isotropic Incidence, $1 \mathrm{MeV}$ Electrons and Space Spectra.}

Isotropic incidence is not calculated by the algorithm in NUMIT. Neither is isotropic data readily available for our purposes. For the isotropic case one may make approximations by modifying the normal incidence data as follows.

Since we are not interested in the details of the electric

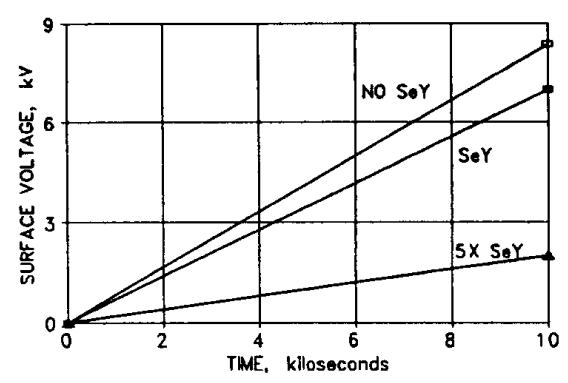

Fig. 4. Surface Voltage by Normally Incident $\mathrm{E}^{-6}$ Spectra.

field distribution within the insulator, we ignore the changes in penetration depth caused by isotropic incidence. This is acceptable for insulators thicker than the penetration range of most of the electrons.

We need to calculate the net current to the insulator, $\mathrm{J}$ (incident) - J(backscatter) - J(secondary electron). Ref. 10 provides the enhancement factors for secondary electrons and backscattered electrons for isotropic spectra relative to normal incidence spectra. The backscatter and secondary electron data for aluminum in Ref. 10 is most applicable to the low atomic number insulating materials. The effect of atomic number on the angular dependence is weak so this comparison adds no error. By integrating over the dependence on incidence angle one finds that isotropic incidence enhances both backscatter and secondary emission by a factor of roughly 3.5 per incident electron relative to normal incidence.

The currents in the isotropic case are estimated as follows. Ref. 14 provides an estimate of the transmission through the cover. Using the isotropic incidence enhancement factor of $\mathbf{3 . 5}$ for the incident electron energies of interest $(100 \mathrm{keV}-10 \mathrm{MeV})$, we may approximate the per electron backscatter yield as $35 \%$ and secondary electron yield as $8 \%$. Thus one approximates the net current to the surface of a thick insulator in the isotropic incidence case. Figs. 5-7 describe the results.

\section{Effect of Surface Currents Flowing to Ground.}

Current can flow in the heavily irradiated and enhanced conductivity regions just below the surface. In theory the surface voltage may be limited by this process. This electron surface current, $J_{S}$, is shown flowing along the surface and "down" the edges to the back plane in Fig. 8. The high energy electron irradiations are scattered at the spacecraft surfaces and can produce a dose along the "sides" of the samples that enhances this leakage path. The geometry of Fig. 8 was extensively used in space [1] and 


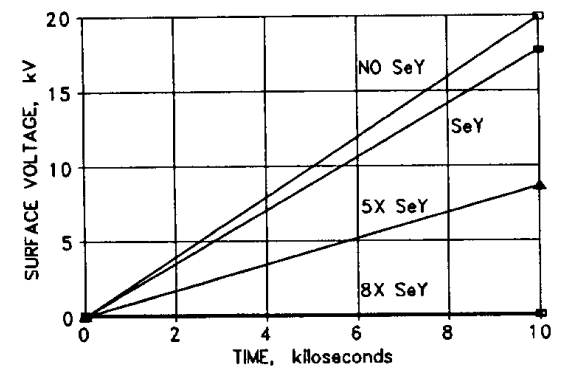

Fig. 5. Surface Voltage by Semi-Isotropic $1 \mathrm{MeV}$ Electrons.

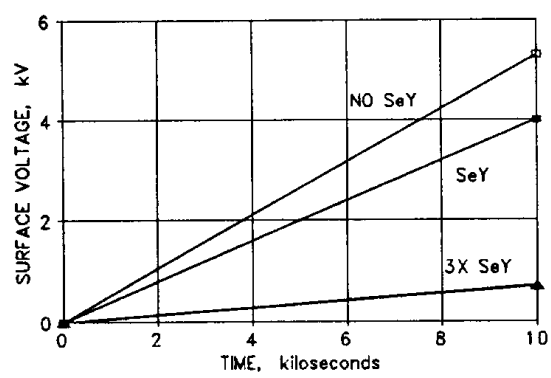

Fig. 6. Surface Voltage by Semi-Isotropic $E^{-4}$ Spectrum.

ground testing [2].

We may perform a simple analytic estimate of this process in steady state by slightly modifying the geometry to that shown in the second half of Fig. 8. The steady state electric field tangent to the surface can be determined for long irradiations by considering current balance. Assume that the surface dose at the edges is the same as at the front surface. This is not a bad assumption considering that in spacecraft the electrons are semi-isotropic. The irradiated region of high conductivity represents the depth of penetration, $R_{e x}$, of the high energy electrons. $W$ is the thickness of the sample and $r_{m}$ is the radius of the sample. Assume thick samples such that $r_{m}>W>2 R_{e x}$; it is only in thick samples that surface leakage dominates over bulk leakage.

In steady state the total current incident on the sample must equal the sum of currents due to electron emission and conduction to ground. Let $\beta$ be the fraction of incident current that escapes the sample as $\mathrm{J}_{\mathrm{T}}+\mathrm{J}_{\mathrm{B}}+\mathrm{SeY}$

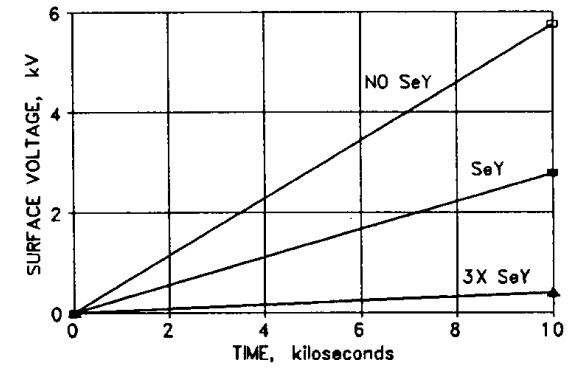

Fig. 7. Surface Voltage by Semi-Isotropic $E^{-6}$ Spectrum.
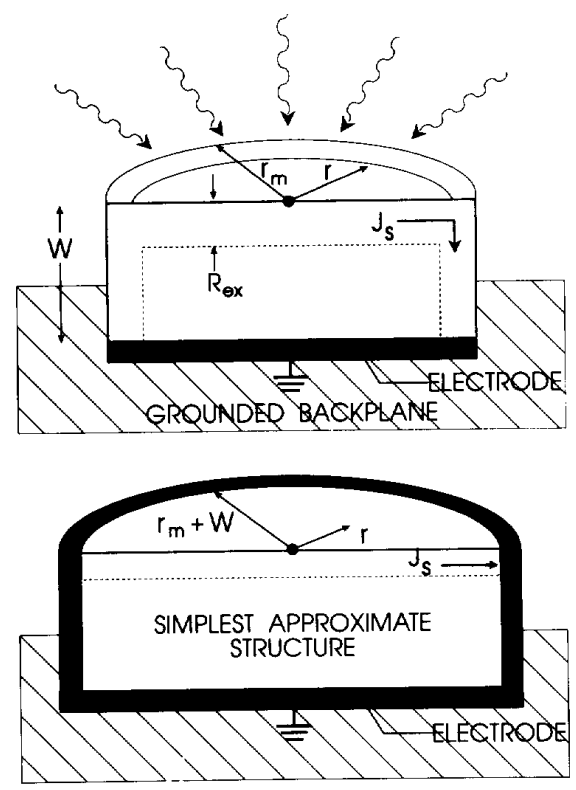

Fig. 8. Geometry for Surface Currents.

(secondary electrons). Then the current which conducts to ground along the surface must be $(1-\beta) \mathrm{AJ}_{\mathrm{I}}$ where $\mathrm{A}$ is the area of the sample.

The position $r$ is measured from the center of the surface of the circular sample. The subsurface radial current density at position $r$ is given by the product of the $r$ component of electric field times the average conductivity of the material in the region delineated by $R_{e x}$,

$$
J_{S}(r)=\sigma E_{r}(r)
$$

Since all the current incident within $r$ must escape in steady state,

$$
J_{S}(r)=(1-\beta) J_{I} \frac{\pi r^{2}}{2 \pi r R_{e x}}
$$


Equating these two forms provides a solution for the electric field in steady state,

$$
\sigma E_{I}=(I-\beta) J_{I} \frac{r}{2 R_{e x}} .
$$

Using averaged conductivity and dose rate, $\mathrm{D}$, we can approximate the conductivity as

$$
\boldsymbol{\sigma}=k_{p} D^{\Delta} .
$$

The exponent $\Delta$ may be approximated to 1.0 , which is its most common value, for ease of calculation by adjusting the term $k_{p}$ to a value appropriate for the particular range of dose rates of interest. Since backscatter and secondary emission remove only a small fraction of the dose, the average dose rate can be related directly to the beam current from the relation: $1 \mathrm{rad} / \mathrm{second}=10^{5}$ watts $/ \mathrm{gram}$. Let $\mathrm{d}$ be density, grams $/ \mathrm{cm}^{3}$.

$$
D=(1-\beta) J_{I} \frac{V_{I}}{d R_{e x}} \times 10^{5}
$$

Combining Eqs. 3-5 provides

$$
E_{r}(r)=\frac{-r d}{2 k_{p} V_{I}} \times 10^{-5} \quad \frac{\text { volts }}{c m} .
$$

Thus $\mathrm{E}_{\mathrm{r}}=0$ at the center and is maximum at the edge where $r=r_{m}+W$.

We can determine the voltage at the center of the structure, $V_{c}$, by integrating $E_{r}(r)$ over the variable $r$.

$$
V_{C}=\frac{-d\left(r_{m}+w\right)^{2}}{4 k_{p} V_{I}} \times 10^{-5} \quad \text { (volts) }
$$

It is especially interesting that the steady state central surface voltage, $V_{c}$, on thick insulators is inversely proportional to the incident electron energy whenever leakage is dominated by sub-surface current to the sample edges. The reason for this is simple: the incidence of higher energy electrons introduces more total conductivity per incident electron than does the incidence of lower

\begin{tabular}{|c|c|c|c|}
\hline$\underline{r}+\underline{W}$ & $=1 \mathrm{~mm}$ & $1 \mathrm{~cm}$ & $10 \mathrm{~cm}$ \\
\hline $\mathrm{V}_{1}=0.1 \mathrm{MeV}$ & $\begin{array}{c}.25 \mathrm{MV} / \mathrm{cm} \\
12 \mathrm{kV}\end{array}$ & $\begin{array}{l}2.5 \mathrm{MV} / \mathrm{cm} \\
1.2 \mathrm{MV} * *\end{array}$ & $\begin{array}{l}25 \mathrm{MV} / \mathrm{cm} \\
120 \mathrm{MV} * *\end{array}$ \\
\hline$V_{1}=1.0 \mathrm{MeV}$ & NA & $\begin{array}{c}.25 \mathrm{MV} / \mathrm{cm} \\
120 \mathrm{kV}\end{array}$ & $\begin{array}{c}2.5 \mathrm{MV} / \mathrm{cm} \\
12 \mathrm{MV} * *\end{array}$ \\
\hline $\mathrm{V}_{\mathrm{I}}=10 . \mathrm{MeV}$ & NA & NA & $\begin{array}{l}.25 \mathrm{MV} / \mathrm{cm} \\
1.2 \mathrm{MV}\end{array}$ \\
\hline
\end{tabular}
energy electrons.

TABLE 1. Steady State $E_{r}$ and $V_{C}$ for Several Sample Sizes and Irradiation Energies, and $k_{p}=2 \times 10^{-17} \mathrm{sec} / \mathrm{ohm}-\mathrm{cm}-\mathrm{rad}$, a Generous Value [11,12].

Table 1 outlines the results for $E_{r}(r+W)$ and $V_{c}$ for typical material parameters and irradiation conditions.
NA means that the model, Eqs. 1-7, is not applicable because $R_{e x}>W$ and therefore leakage currents through the bulk of the insulator will dominate and hold the surface voltage to lower values than predicted by Eq. 7. ** means that the center point voltage approaches or exceeds $V_{I}$, so the model fails to predict steady state values properly yet correctly indicates that high voltages are attained.

The data along the diagonal gives us the best sense of how surface leakage affects the surface electric field and the central surface voltage. The strength of $E_{r}$ will approach or exceed $250 \mathrm{keV} / \mathrm{cm}$ and the maximum surface voltage will be at least ten percent of the incident beam voltage. The sample must be thick for this to hold true.

The most important results of this study of sub-surface conduction phenomena are:

1. The electric field near the edge of the thick sample is strongly enhanced as the size of the sample is increased. 2. For typical space spectra the surface voltage will not be held by this sub-surface conduction phenomena to safe levels unless the size of the sample is below $1 \mathrm{~mm}$.

There is another effect discussed by Reeves and Balmain that also enhances the electric field strength at the edge of the sample [13]. As the sample charges up, its surface voltage deflects incoming electrons so that they preferentially impact the sample at its edges and thus induce extra charging there. With the monoenergetic normalincidence multi-keV beams used in their study, this effect achieved surface fields as high as $10^{6} \mathrm{~V} / \mathrm{cm}$. However, under high energy space spectra this deflection effect is not as important as it is for the cases [13] under 10 or $20 \mathrm{keV}$ bombardment.

Secondary electron emission is affected by the tangential surface electric field. This process further enhances the field strength at the edge of the samples. The most negative region at the center of the sample will retard secondary electron emission from the edges of the sample. The electric field in the vacuum space is produced not only by the electrons trapped in the insulator, it is also modified by the image charges in the nearby conductors. The net result is that strongly charged surfaces often create an electric field at weakly charged insulator surfaces that retards secondary electron emission from these surfaces. Thus, the edges of the samples will emit fewer electrons and become more highly charged until a balance is attained.

\section{E. Arbitrary Solar Blanket Thickness.}

The previous calculations have been performed for a specific solar blanket thickness. We can investigate the effect of changing the solar blanket thickness in a simple way. It turns out that the generic charging problem remains for any reasonable thickness blanket including some shielding.

The HE electrons pass through the solar blanket, through the vacuum space and finally into the insulator. We are 
interested in the currents flowing across the vacuum space. If the secondary electron yield from the insulator exceeds the HE electron current to the insulator, then no surface charging will occur. We can perform the computer calculations while assuming a vacuum gap at any depth in the slab geometry. The vacuum gap does not perturb the HE electrons in any way for our 1-D problem.

The NUMIT code provides both the HE electron current and the dose rate at all depths in the slab geometry. We may determine the available secondary electron current at any depth by multiplying the dose rate by the nominal secondary electron yield factor discussed above, $1 \times 10^{-13}$. Insulator charging will occur wherever the available secondary electron current is less than the HE current.

Fig. 9 is a plot of the ratio of secondary electron current to HE electron current as a function of depth for three cases of normal incidence. The power law space spectra never develops the no-charging situation at any depth. Fig. 9 indicates that any thickness solar blanket will maintain the charging condition as long as the incident radiation is sufficient to overcome dark conductivity leakage.

Secondary electron emission alone will not prevent surface charging at any depth inside a satellite. This basic conclusion is not changed in the isotropic irradiation case even though the ratio is somewhat less. The $1 \mathrm{MeV}$ case shows that in ground tests there may occur a situation where less charging is observed if the insulator is located exactly at the end of range. This situation is of little importance and can be avoided.

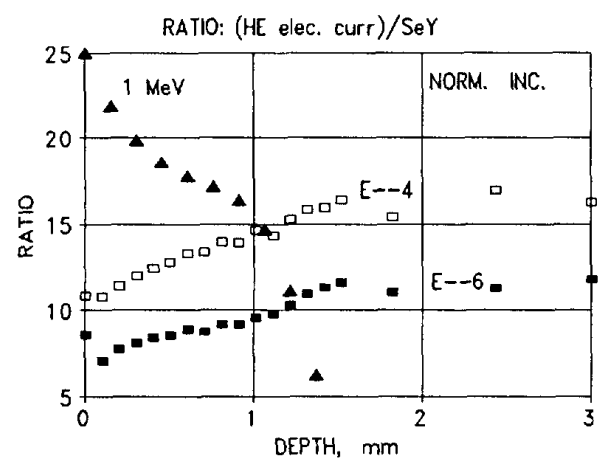

Fig. 9. Ratio of High Energy Electron Current to Secondary Electron current as a Function of Thickness of Solar Blanket.

Fig. 10 helps one to estimate whether the thicker solar blanket has attenuated the HE electron current sufficiently to allow dark conductivity leakage to prevent charging. The use of this graph is straightforward and needs no discussion.

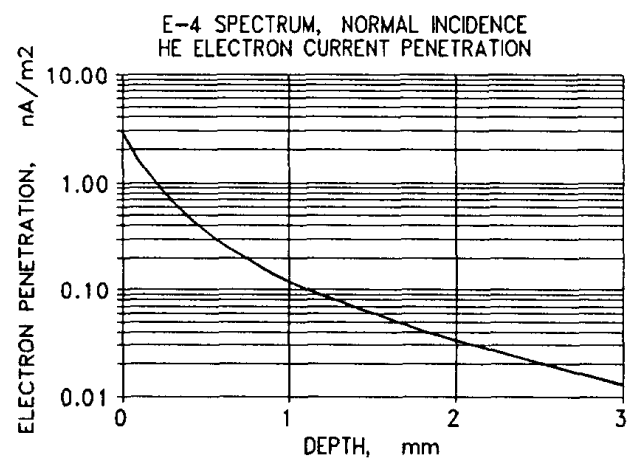

Fig. 10. HE Current Penetration Beyond The $0.4 \mathrm{~mm}$ Blanket for Normal Incidence $E^{-4}$ Spectrum at $1 \mathrm{pA} / \mathrm{cm}^{2}$.

\section{F. Effect of Bulk Leakage in Thin Insulators with Grounded Back Surfaces.}

NUMIT directly provides an estimate of the surface voltage as a function of sample thickness for the case where the back side of the sample is grounded. A common example of a thin insulator is thermal control paint. Fig. 11 shows how high the voltage can become at long times for thin insulators. We assume the same level of dark conductivity and RIC as before. Additionally we assume a factor ten increase in dark conductivity because thin layers, especially with an electroded surface, often have higher dark conductivities. We discover that the steady state voltage is held low only for very thin insulators with the rear surface in good contact with the spacecraft frame. These estimates are for a large area sample in which surface leakage is not an issue.

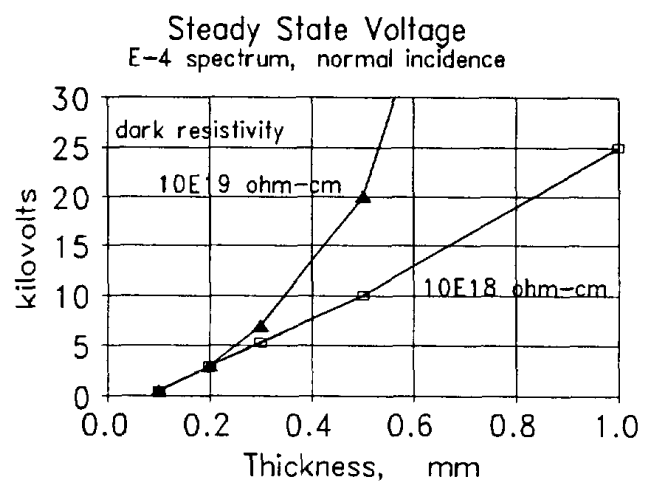

Fig. 11. Surface Voltage on Thin Samples at Long Times. 


\section{G. Neutralizing Current From Space Plasma.}

Small holes in the thermal control blanket are capable of passing sufficient ions from the ambient plasma to neutralize the surface potential at low altitudes. The plasma ions have velocities comparable to spacecraft velocities. The current collected through a hole depends on the orientation of the hole w.r.t. the spacecraft velocity vector. Ion densities vary from $10^{6}$ to $10^{0} \mathrm{~cm}^{-3}$ [15]. Satellite velocities are of the order $10^{6} \mathrm{~cm} / \mathrm{sec}$. Therefore a properly oriented hole will collect on the order of $10^{-6}$ to $10^{-14} \mathrm{~A} / \mathrm{cm}^{2}$ depending on both orbital velocity and plasma density which, in turn, depends primarily on altitude and latitude.

The CRRES/IDM samples had a $1 \mathrm{~mm}^{2}$ hole for evacuating its samples. These holes could have collected an estimated $10^{-10}$ Amperes at CRRES's lowest altitude and thereby affected the charging of the IDM samples on some orbits. This has yet to be carefully investigated.

\section{COMMENT}

Our focus has been on surface voltages in spacecraft cavities. High surface voltages are not necessarily a problem by themselves. The problems occur when a small spontaneous discharge of plasma and gas neutrals is produced in the cavity. We do not address the cause of the initial discharge. But once a discharge has begun, the high surface voltages, acting on the gas and plasma, will cause a rapid avalanching of charge and current in the cavity [4]. The resulting pulsed currents produce electromagnetic interference with the onboard electronics. High surface voltages should be avoided.

\section{CONCLUSIONS}

Insulating surfaces inside closed spacecraft cavities will often charge to extremely high voltages when leakage is not provided. The surfaces of insulated wires in cable bundles which are wrapped in metalized foil will charge to high voltages internal to the foil wrap. Discharges would occur internal to the foil shield. The insulator surfaces should be guarded (shielded) from exposure to the environment of the spacecraft cavities.

Openings to allow the collection of space plasma in low orbits would provide leakage for small insulators.

Electrically leaky insulating materials should be chosen for spacecraft insulation. The conductivity should be determined for the space environment: a long duration exposure to vacuum and radiation.

The insulators should be made as thin and as small as possible. Insulators smaller than $0.1 \mathrm{~mm}$ in dimension have inherent properties which make them much safer.

Heavier radiation shielding only helps when the current density penetrating the shield is so low that the dark leakage of the insulator bleeds off the voltage. Usually, such shielding is impractical.

Surface potential monitors should be flown in space to investigate the effect by monitoring the potential inside the spacecraft.

\section{REFERENCES}

1. A. R. Frederickson, E. G. Holeman and E. G. Mullen, "Characteristics of Spontaneous Electrical Discharging of

Various Insulators in Space Radiations," IEEE Trans. Nuc. Sci. 39, 1773-84, Dec., 1992. (See section on electric fields.)

2. P. G. Coakley, M. J. Treadaway and P. A. Robinson, "Low Flux Laboratory Test of the Internal Discharge Monitor (IDM) Experiment Intended for CRRES, " IEEE Trans. Nuc. Sci. 32, 4066-72, 1985.

3. K. G. Balmain and G. R. Dubois, "Surface Discharges on Teflon, Mylar and Kapton," IEEE Trans. Nuc. Sci. $\underline{26}$, 5146-51, 1979.

4. A. R. Frederickson, Leon Levy and C. L. Enloe, "Radiation-induced Electrical Discharges in Complex Structures," IEEE Trans. Electrical Insulation 27, 1166-78, Dec. 1992.

5. I. Katz et al, "The Capabilities of the NASA Charging Analyzer Program," in Spacecraft Charging Technology 1978, NASA CP-2071 or AFGL-TR-79-0082, p 101, 1979. NTIS AD/A084626.

6. A. R. Frederickson, "Electric Discharge Pulses in Irradiated Solid Dielectrics in Space," IEEE Trans. Electrical Insulation 18, 337-49, June, 1983.

7. M. Hikita, M. Zahn, K. A. Wright, C. M. Cooke and J. Brennan, "Kerr Electro-optic Field Mapping Measurements in Electron-beam Irradiated Polymethylmethacrylate, " IEEE Trans. Electrical Insulation 23, 861-80, Oct. 1988.

8. E. A. Burke, "Secondary Emission from Polymers," IEEE Trans. Nuc. Sci. 27, 1760-4, Dec., 1980.

9. E. A. Burke, J. A. Wall and A. R. Frederickson, "Radiation-induced Low Energy Electron Emission from Metals," IEEE Trans. Nuc. Sci. 17, 193-9, Dec., 1970. 10. A. R. Frederickson and E. A. Burke, "Total Electron Backscatter and Backemission Yields from Metals Bombarded at Several Angles by 0.4 to $1.4 \mathrm{MeV}$ Electrons," IEEE Trans. Nuc. Sci. 19 No. 6, Dec. 1972 , 160-6.

11. J. F. Fowler, Proceedings Royal Soc. A236, 464-80, 1956.

12. A. R. Frederickson, "Radiation Induced Currents and Conductivity in Dielectrics, " IEEE Trans. Nuc. Sci. 24, No. 6., Dec, 1976, 2532-9.

13. R. D. Reeves and K. G. Balmain, "Two-dimensional Electron Beam Charging Model for Polymer Films, " IEEE Trans. Nuc. Sci. 28 , No. 6, Dec. 1981, 4547-52.

14. A. R. Frederickson, E. G. Mullen, K. J. Kerns, P. A. Robinson and E. G. Holeman, "The CRRES IDM Spacecraft Experiment For Insulator Discharge Pulses," IEEE Trans. Nuc. Sci. 40, No. 2, April 1993, 233-41.

15. Handbook of Geophysics and the Space Environment, Air Force Geophysics Laboratory, U. S. Air Force, 1985. Available from the NTIS, Acession Number ADA 167000. 